\title{
Simulation of swelling of model polymeric gels by subcritical and supercritical solvents
}

\author{
Fernando A. Escobedo a) and Juan J. de Pablo \\ Department of Chemical Engineering, University of Wisconsin-Madison, Madison, Wisconsin 53706-1691
}

(Received 17 July 1998; accepted 18 September 1998)

\begin{abstract}
Monte Carlo simulations have been performed to examine the volumetric behavior of model nonionic gel systems where the solvent is at subcritical and supercritical conditions. It is found that the degree of swelling correlates well with the density changes of the solvent: swelling decreases as the solvent becomes less dense. As the temperature is increased at constant pressure, gel swelling exhibits a monotonic decreasing trend whose onset approximately agrees with the solvent's boiling point for subcritical pressures and, for supercritical pressure, with the location of the lower critical solution temperature (LCST) of the solvent-uncrosslinked polymer system. Evidence of an incipient first-order volume phase transition has been detected for polymeric networks with long strand lengths in contact with a supercritical solvent. (C) 1999 American Institute of Physics.

[S0021-9606(98)51448-1]
\end{abstract}

\section{INTRODUCTION}

When linear or branched polymeric chains with polyfunctional sites react in the proper environment, they can form complex crosslinked structures known as polymeric networks. Linear chain sections connecting two crosslinking sites are termed active strands. Active strands limit the ability of a polymeric network to absorb a solvent by creating an elastic pressure that opposes the swelling of the resulting gel (and balances the osmotic pressure). ${ }^{1}$ Polymer gels have important applications as absorbent materials and separation agents in various industries. To a large extent, the ultimate purpose of a gel is that of absorbing and releasing (selectively) some substances from a fluid medium. The key for development and use of "intelligent" gels therefore resides in our ability to control these absorption and release processes, e.g., the degree of gel swelling. ${ }^{2}$ Although studies of gel swelling have been confined to conditions where the solvent is subcritical, use of supercritical solvents can provide new opportunities for development of novel separation technologies. Supercritical fluids are used in extraction processes due to the ease with which their solvent quality can be tuned by small changes in pressure and temperature; it is therefore of interest to explore the possible advantages of separation processes comprising both a supercritical solvent and a polymeric gel.

The physical similarity between a polymer solution (in a single solvent) and a swollen gel led previous researchers to predict discontinuous volume phase transitions in gels in analogy to the coil-globule transition of polymers in solution. ${ }^{3}$ Theoretical and experimental work has confirmed these predictions and, in fact, some systems have been shown to exhibit multiple phase transitions. ${ }^{2}$ It has been shown that volume phase transitions can be generated by changes in composition of the solvent, temperature, pressure,

\footnotetext{
${ }^{a)}$ Present address: School of Chemical Engineering, Cornell University, Ithaca, NY 14853.
}

$\mathrm{pH}$, ionic concentration, stress, electric fields, etc. The fact that a small change in a controllable variable of the system can generate large changes of its extensive properties (e.g., volume) is a feature of great interest for technological applications. Discontinuous volume phase changes are generally favored by long polymers (strand length), large persistence length, and extensive ionization. ${ }^{2}$

Temperature sensitive gels are of particular interest for chemical separations because they are usually un-affected by changes in $\mathrm{pH}$ or ionic strength. A wellstudied example of a temperature sensitive gel is provided by poly(N-isopropylacrylamide) (NIPA) in water. $^{4-6}$ This gel is highly swollen at low temperatures and shrinks as the temperature increases. The driving force underlying the volume phase transition that these gels exhibit is a lower critical solution temperature (LCST). Below the LCST, uncrosslinked NIPA chains are completely miscible with water; above the LCST, a polymer-rich and a water-rich phase are formed. This change in the character of polymer-solvent miscibility that occurs at the LCST is manifest in a gel as variations in the amount of absorbed solvent; above the LCST the gel tends to collapse and forms a shrunken, "polymer-rich", phase.

The LCST in the NIPA-water system occurs at temperatures well below the critical temperature of water $\left(305-320 \mathrm{~K} \ll T_{c}=647 \mathrm{~K}\right)$ and both phases exhibit liquidlike densities; it is therefore associated with a liquid-liquid phase equilibrium (LLE). This type of LLE-LCST is caused by the effect of temperature (and density) on hydrogen bond associations between water and amide groups of the polymer. Note, however, that not all nonionic polymer-solvent systems that exhibit a LCST give rise to a first-order transition in gel volume with temperature. ${ }^{7}$ For example, linear poly(diethylacrylamide) in water ${ }^{6}$ does exhibit a LCST at atmospheric pressure, but gels prepared from this polymer do not exhibit a discontinuous volume phase transition. ${ }^{6}$ The different behavior of this system and the NIPA gel arises from 


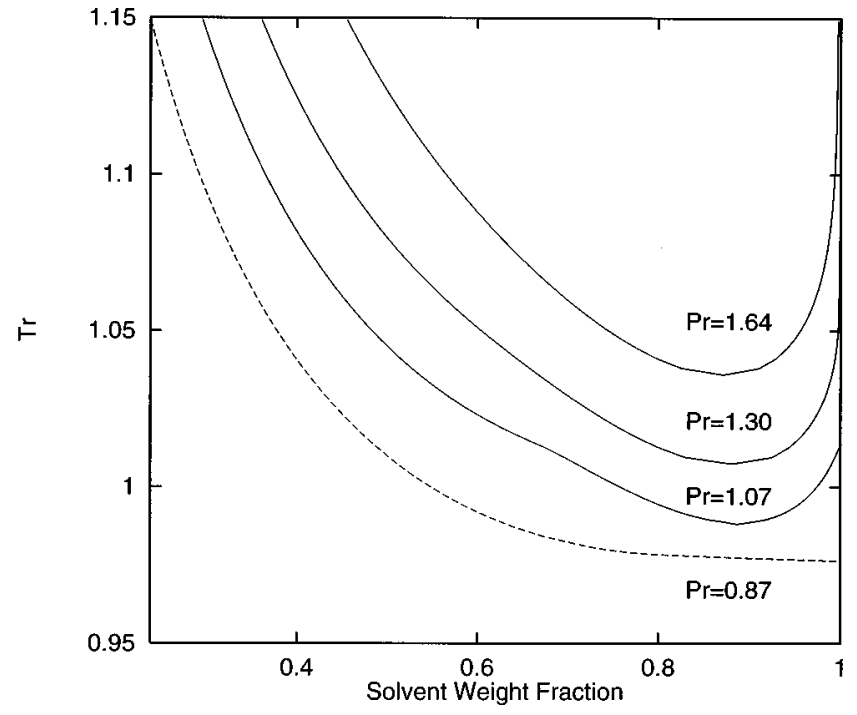

FIG. 1. Qualitative effect of pressure on the miscibility of a binary mixture of polymer and solvent. Curves have been calculated with the SanchezLacombe theory (Refs. 13 and 14) for a mixture of ethane (solvent) and heptadecane (short polymer). Full curves correspond to supercritical pressures and dashed lines to subcritical pressures [reduced values with respect to the calculated critical properties of the solvent $\left(T_{c}=315 \mathrm{~K}, P_{c}\right.$ $=57.6 \mathrm{~atm})]$. Similar results can be observed by employing a cubic equation of state.

small differences in solvent-polymer energetic interactions and crosslinking density.

Some nonpolar polymer-solvent systems exhibit a LCST at temperatures and pressures slightly above the critical point of the solvent. ${ }^{8}$ We will refer to this type of behavior as a vapor-liquid equilibrium (VLE) type of LCST, because the immiscibility is essentially caused by the density difference between the pure components; the polymer is dense, liquidlike, and the solvent is light, vaporlike. As shown in Fig. 1, a VLE-LCST can also occur in a system of polymer and subcritical solvent; in this case, the LCST essentially corresponds to the boiling temperature of the pure solvent at the given (subcritical) pressure. If the solvent expands much faster than the polymer upon increasing temperature (and gradually becomes a vaporlike, poorer solvent), the solution will eventually phase separate into a solvent-rich and a polymer-rich phase. Experimental ${ }^{9}$ and simulation ${ }^{10}$ evidence indicate that, while chain length decreases the LCST (slightly), pressure has the opposite effect. Further, if high enough, pressure can entirely suppress phase separation. ${ }^{11}$

It is unclear, however, whether a VLE-LCST could drive a volume phase transition in the corresponding solvent-gel system or not. If such transitions exist, it would be of interest to determine what factors determine their order. If the solvent is at a subcritical pressure, for example, solventpolymer interactions in the gel will bear some resemblance to those in a fluid-porous solid system; however, an abrupt fluid desorption from a solid can not be unambiguously translated into a discontinuous transition in gel swelling.

While several analytical theories are available to describe either supercritical vapor-liquid equilibria or swelling of (usually ionic) gels at low temperatures, there is a severe lack of models capable of describing the gel-swelling behavior induced by supercritical fluids. A theory of gel swelling by a subcritical or a supercritical solvent must take into account the effects of mixing, elasticity, and compressibility on the properties of the system. Within the framework of Flory-Huggins' lattice theory of polymer-solvent thermodynamics, ${ }^{12}$ the effect of compressibility can be conveniently included by introducing holes or empty sites in the lattice. Such an approach was employed by Sanchez and Lacombe in their lattice-fluid theory, which has been shown to provide a satisfactory description of different types of phase equilibria in mixtures containing macromolecules, including LCST-type of behavior. ${ }^{13}$ For incompressible systems, one of the most widely used mean-field theories to describe the swelling of gels is that of Flory and Rehner. ${ }^{12}$ The theory proposed by Marchetti et al..$^{5}$ for gel swelling is a simple combination of the Sanchez-Lacombe lattice theory for the mixing thermodynamics of compressible polymersolvent systems ${ }^{13,14}$ and the Flory-Rehner theory for the elastic free energy of a network. ${ }^{12}$ Marchetti et al.'s theory has several adjustable parameters which can be fitted to select experimental data; however, the extrapolations of such a model are only reliable over short ranges of state variables (e.g., temperature). ${ }^{6}$ The limited predictive capabilities of Marchetti et al.'s theory are due to the inaccuracies associated with the assumptions of both the fluid-lattice theory and the Flory-Rehner theory (the additivity of elastic contributions and the Gaussian behavior of elastic chains).

In this work we study by molecular simulation a simple, well-characterized nonpolar gel consisting of a near-critical spherical solvent and a flexible crosslinked polymer of perfect topology. In this way, we can isolate the relationship between the presence of a VLE-LCST in the solventpolymer system and volume phase transitions in the gel. In the following section we describe the coarse-grained molecular models adopted in this work and the Monte Carlo methods employed to simulate them. Subsequent sections are devoted to a discussion of our results and conclusions.

\section{METHODOLOGY}

The molecular model adopted in this study is consistent with that examined in some of our earlier work. ${ }^{15,16}$ All network strands have the same length, $N_{\text {str }}$, and are interconnected by tetrafunctional sites. Periodic boundary conditions are employed to emulate an infinite system. Such perfect crosslinking purposely neglects the effect of topological defects while providing an approximate description of gel elasticity. To account for compressibility effects on swelling, molecular sites are allowed to move freely in a continuum. To ensure that the structure of the gel and solvent resembles that of real fluids, interaction sites must have a repulsive core and an attractive shell. In this work we model such interactions in two ways. We use a square-well (SW) potential energy function,

$$
\begin{aligned}
u_{\mathrm{SW}}(r) & =\infty, \quad r<\lambda_{o v} \sigma \\
& =-\epsilon, \quad \lambda_{o v} \sigma \leqslant r \leqslant \lambda_{\mathrm{cut}} \sigma \\
& =0, \quad r>\lambda_{\mathrm{cut}} \sigma,
\end{aligned}
$$


and a modified cut-and-shifted Lennard-Jones (MLJ) potential energy function,

$$
\begin{aligned}
u_{\mathrm{MLJ}}(r) & =\infty, \quad r<\lambda_{o v} \sigma \\
& =F_{\mathrm{LJ}}(r)-F_{\mathrm{LJ}}\left(\lambda_{\mathrm{cut}} \sigma\right), \quad \lambda_{o v} \sigma \leqslant r \leqslant \lambda_{\mathrm{cut}} \sigma \\
& =0, \quad r>\lambda_{\mathrm{cut}} \sigma,
\end{aligned}
$$

where

$$
F_{\mathrm{LJ}}(x)=4 \epsilon\left[\left(\frac{\sigma}{x}\right)^{12}-\left[\left(\frac{\sigma}{x}\right)^{6}\right] .\right.
$$

For SW sites we use $\lambda_{o v}=1$ and $\lambda_{\text {cut }}=1.5$. For MLJ sites, however, we use $\lambda_{o v}=0.96$ and $\lambda_{\text {cut }}=1.57$. These values of $\lambda_{o v}$ and $\lambda_{\text {cut }}$ for MLJ sites were selected based on purely computational grounds; they increase significantly the efficiency of volume fluctuations, which tend to be particularly sluggish in polymeric gel systems. As discussed later, for this MLJ potential we are able to achieve higher resolution than for the SW model using less computer time. Sites belonging to solvent or polymeric species experience the same interactions; the same values of $\sigma, \epsilon$, and $\lambda$ are used for likeand unlike-species interactions.

Chain molecules and network strands are modeled as a collection of tangent sites; the bond length was fixed at $\lambda \sigma$ for both SW and MLJ systems. Network strands of 10, 20 , and 40 sites long were employed for our simulations (note that a single site is meant to represent several repeat units of a polymer). Two system sizes were considered for the polymeric network: one consisted of 16 crosslinking sites and 32 strands, and the other was four times larger. The larger system was simulated only to confirm results for the smaller system at a few select state points.

Intramolecular rearrangements of polymeric sites were performed by means of extended continuum configurational moves $;{ }^{17}$ volume changes were carried out by means of cluster moves. ${ }^{15}$ Most simulations of gel swelling were conducted in a Grand canonical type of ensemble (or, more properly, an osmotic ensemble), in which the number of solvent molecules is allowed to fluctuate in response to an imposed chemical potential, and the volume of the gel changes in response to an imposed pressure. The properties of the pure solvent (PVT and chemical potential) were obtained from standard isothermal-isobaric simulations.

A gel-swelling run consisted of $10^{6}-10^{7}$ cycles (for a given temperature, pressure, and a precalculated solvent chemical potential). Each cycle consisted of one volume move attempt (on average), one molecular rearrangement attempt per site in the system, and one molecular insertion per solvent molecule. A gel-swelling run was repeated until convergence in density and composition was attained. Longer runs were usually necessary for highly swollen (long strand) gels, where the total number of sites is $O\left(10^{4}\right)$; in these cases, simulation of a single state point can require from one to two weeks on a workstation with an Alpha $533 \mathrm{MHz}$ processor.

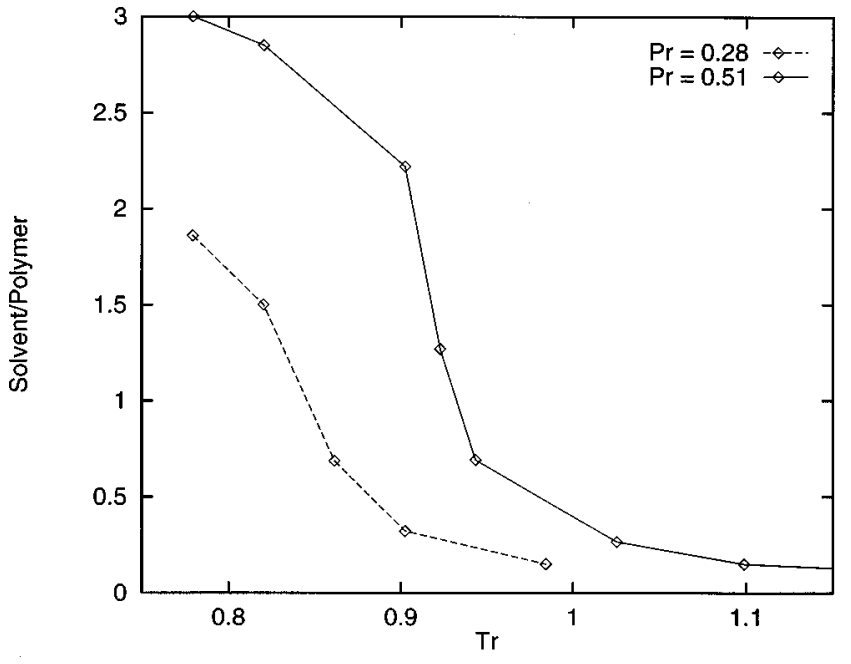

FIG. 2. Subcritical isobars for the swelling of SW gels as a function of reduced temperature. Temperature and pressure are reduced with respect to the critical properties of the pure SW fluid. The ordinate is the ratio of the number of solvent sites over the number of network sites in the gel. The standard deviation of each point is approximately three times the size of the symbols.

\section{RESULTS}

Figure 2 shows the degree of swelling of a ten-site strand network (in terms of sites of solvent absorbed per site of " dry" polymer network) as a function of reduced temperature for two subcritical pressures. The gel is in equilibrium with a SW monomeric solvent. Temperatures and pressures are reduced with respect to the critical properties of the pure SW solvent, e.g., $T_{c}^{*}=k_{B} T_{c} / \epsilon=1.219$ and $P_{c}^{*}=P_{c} \sigma^{3} / \epsilon$ $=0.108 .{ }^{18}$ The boiling point of the solvent is $T_{b_{r}}=0.845$ at $P_{r}=0.278$, and $T_{b_{r}}=0.919$ at $P_{r}=0.509$. For both isobars, swelling exhibits a rapid decline near the boiling point. While gel shrinkage is mild before $T_{b}$ and much more pronounced after $T_{b}$, the swelling transition appears to be gradual. As the temperature increases well above $T_{b}$, the solvent becomes a dilute vapor and swelling approaches a vanishingly small value. The variations of the amount of solvent in the gel shown in Fig. 2 bear some resemblance to those of a fluid that is being desorbed from a porous solid.

Figure 3 shows results for the same system as in Fig. 2, but at supercritical pressures. In this case there is not a boiling point that could be used to predict the onset of gel collapse. Swelling decreases rapidly as the system approaches the critical temperature of the solvent, where it appears to reach a plateau (that spans a short temperature range) and then decreases again. The temperature where this plateau ends increases with pressure. For the two lowest pressures, such a plateau end point is marked by the presence of a small peak; in view of the large uncertainty of the data and the presence of important finite size effects, however, the existence of the peak cannot be unambiguously established.

Figure 4(a) shows the variations of the reduced isothermal compressibility $\left(\kappa_{T_{r}}\right.$ with temperature for the pure SW monomer solvent). The symbols correspond to results of our simulations. For comparison, predictions from a secondorder perturbation equation of state $(\mathrm{EoS})^{19}$ are also shown. Although the agreement between simulation and theory is not quantitative, the EoS is able to capture the main features 


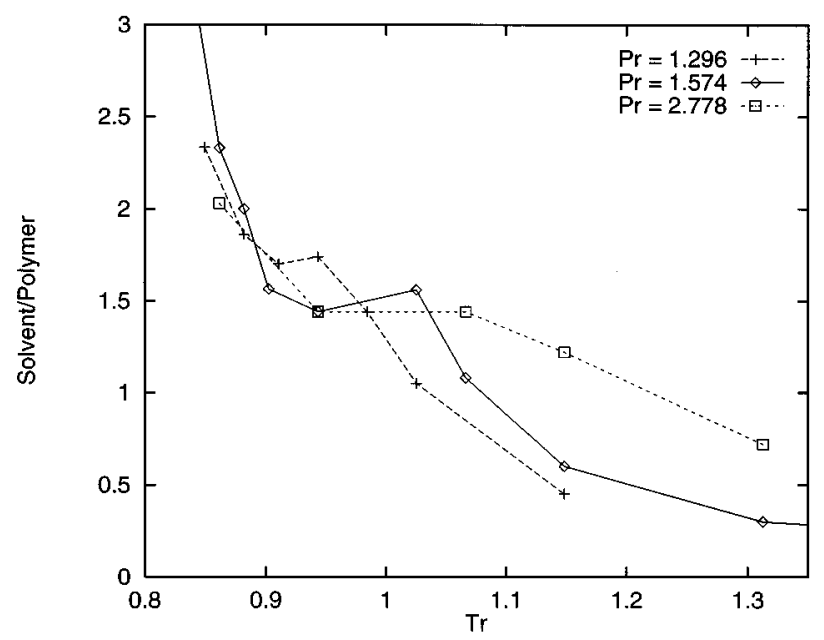

FIG. 3. Supercritical isobars for the swelling of SW gels as a function of reduced temperature. Temperature and pressure are reduced with respect to the critical properties of the pure SW fluid. The standard deviation of a simulation point is typically three to four times the size of the symbol. See also the legend of Fig. 1.

of $\kappa_{T_{r}}$; the peak in $\kappa_{T_{r}}$ is higher for supercritical pressures closer to unity. Figure 4(b) shows additional curves obtained from the EoS; $\kappa_{T_{r}}$ exhibits a discontinuity at the liquid to vapor transition (from low to high $\kappa_{T_{r}}$ ). For $P_{r}<1$, as pressure increases, the peak in $\kappa_{T_{r}}$ becomes more pronounced; $\kappa_{T_{r}}$ diverges at the critical point. For $P_{r}>1$, the discontinuity in $\kappa_{T_{r}}$ disappears but the general appearance of the curve is maintained in the vicinity of the critical pressure; as $P_{r}$ becomes much greater than unity, the peak is smoothed out and eventually disappears.

Two general conclusions can be drawn from the results of Figs. 2 through 4: (i) at any given temperature, swelling is generally greater for higher pressures, which correlates well with increasing values of the pure solvent density, and (ii) a fast reduction of swelling with increasing temperature is associated with the existence of a peak in the isothermal compressibility of the pure fluid. Experimental evidence in support of point (i) has been reported in the past for subcritical gels $^{20}$ and, theoretically, for athermal gels. ${ }^{15}$ Figure 5 provides a phenomenological basis for this point: several isobars of packing fraction versus temperature are shown for the pure SW solvent at different sub- and supercritical pressures and for the polymeric network at zero pressure. ${ }^{21}$ As the gel and the coexisting solvent are compressed (at a given temperature), their density increases at the expense of a reduction of free volume. The increase in solvent density with pressure is more pronounced for temperatures near and above the critical point (due to the large compressibility of the fluid). Solvent-polymer solubility or, more precisely, gel swelling, will generally be higher at elevated pressures because the difference in density (or free volume) between the pure solvent and the pure network becomes smaller. The denser the solvent, the more effective it is in screening intrachain forces. It can also be inferred that changes in gel swelling will be more pronounced when the difference in density between pure solvent and pure network varies more rapidly;

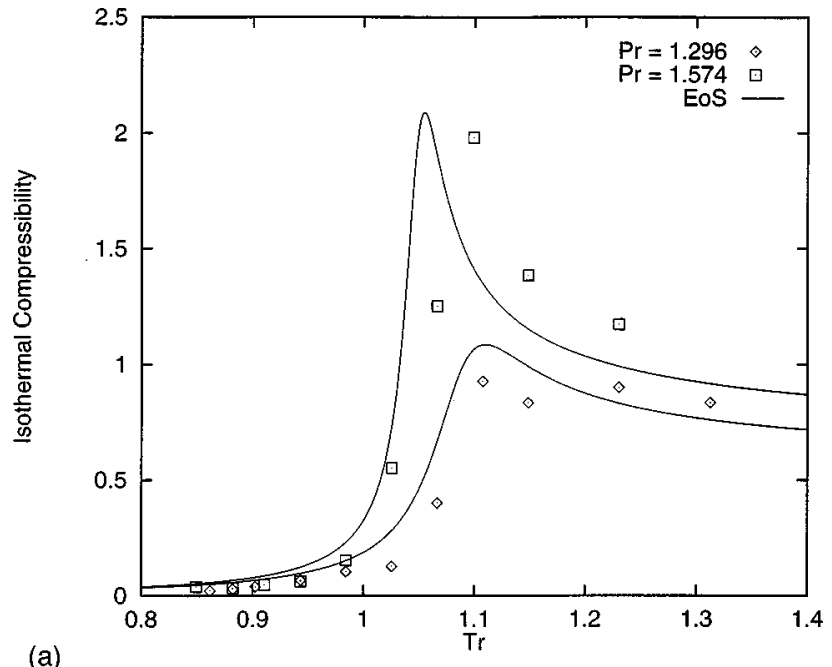

(a)

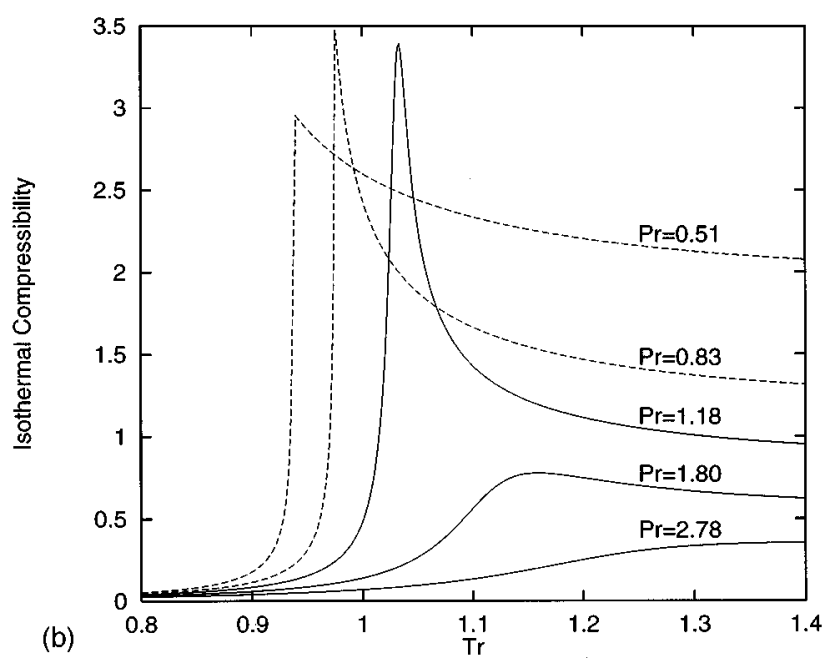

FIG. 4. Isothermal compressibility of the SW monomeric fluid as a function of reduced temperature and reduced pressure. The symbols in (a) correspond to simulation data. The lines in (a) and (b) are the predictions of an equation of state derived from perturbation theory (Ref. 19). The isothermal compressibility is given in reduced units (properties are reduced with respect to $T_{c}$ and $P_{c}$ of the pure SW fluid).

that happens at $T_{r}=T_{b_{r}}$ for $P_{r}<1$ and near $T_{r}=1$ for $P_{r}>1$.

The effects of pressure and temperature on polymersolvent affinity discussed in the previous paragraph are also relevant for a system of solvent and uncrosslinked polymer. In fact, nonpolar solvent-polymer systems usually exhibit complete miscibility for low temperatures and dense solvents, and partial miscibility for higher temperatures and lighter solvents. At constant pressure, the onset of partial miscibility upon heating is marked by a LCST. This is illustrated in Fig. 6 for square-well systems at $P_{r}=1.57$. The LCST lies around $T_{r}=1.12$ for a monomer-16-mer system and at $T_{r}=1.07$ for a monomer-42-mer system, respectively. As found in previous experimental and theoretical studies, ${ }^{8,10}$ a lower LCST is associated with longer chains. The issue of whether or not there is an asymptotic limiting value, $T_{\infty-\text { mer }}$, for the LCST of an infinitely long chain, however, has not been fully resolved. This issue is relevant for gel swelling, as the volumetric behavior of a gel should be more akin to that 


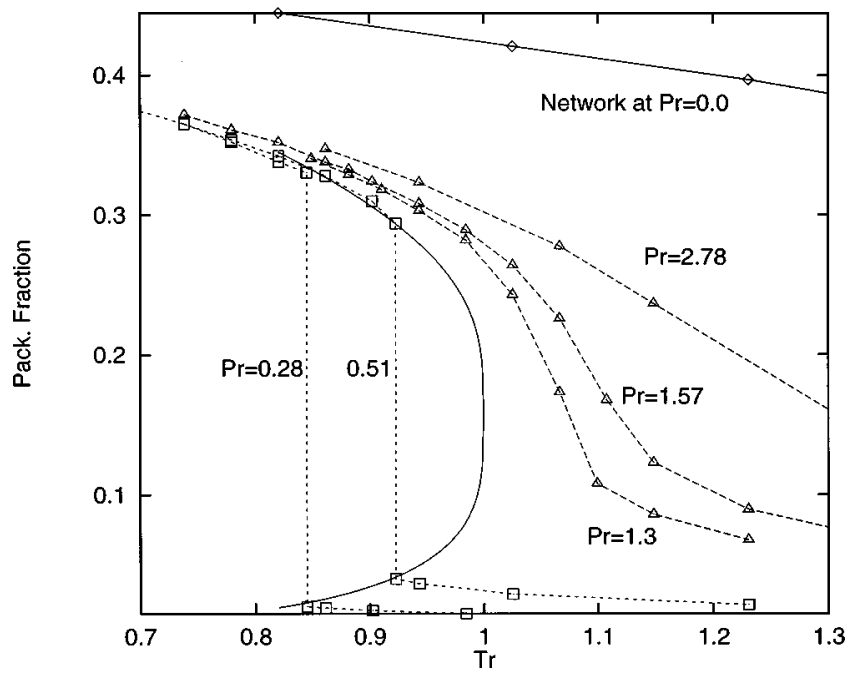

FIG. 5. Simulated sub- and supercritical isobars for a pure square-well monomer (squares and triangles). The full line around the critical point is a fit to simulated vapor-liquid coexistence data from Ref. 18. The upper curve and diamonds correspond to a pure ten-site strand network at $P_{r}$ $=0.0$. Standard deviations are typically the same size as the symbols.

of a solvent-polymer system when the polymer chains are very long. It should be noted that the theory of Sanchez and Lacombe $^{14}$ does predict an asymptotic finite value for $T_{\infty}-$ mer .

An approximate method for locating the LCST relevant for a crosslinked polymer is provided by simulation of a monomer-polymer system, in which the polymer has a compressibility as close as possible to that of the pure crosslinked polymer. This requirement can be best fulfilled by a polymer with the same connectivity ratio as that of the crosslinked polymer ${ }^{15}$ (that is, the same ratio of covalent bonds to sites in the system). Figure 6 shows the simulated data for one such polymer, a 42-mer with intramolecular crosslinking (a so-called "pumpkin" molecule because it is

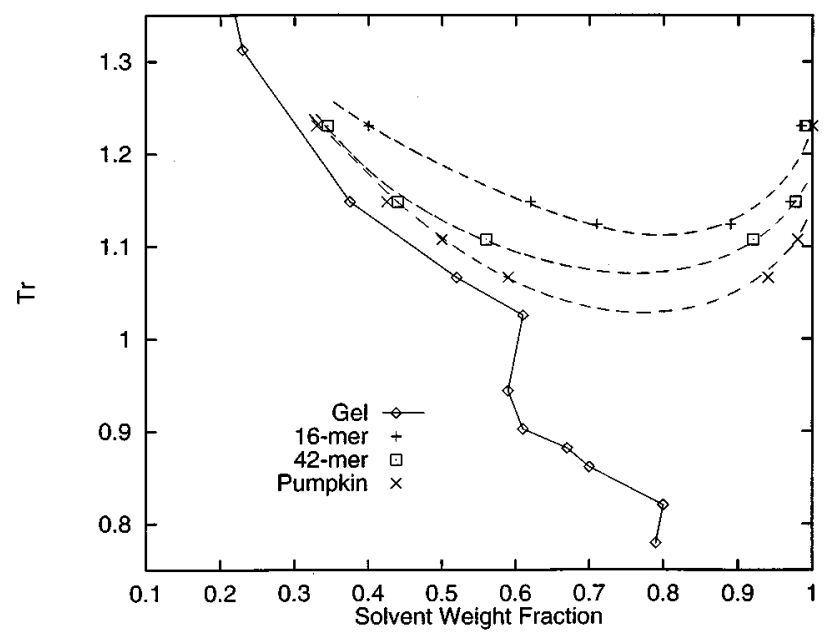

FIG. 6. Simulated isobars $\left(P_{r}=1.57\right)$ of gel swelling and polymer-solvent miscibility for SW systems. The upper curves represent the coexistence compositions of mixtures of solvent with 16-mer (triangles), 42-mers (crosses), and pumpkin molecules (squares), respectively; the dashed lines are a guide to the eye. The diamonds and the full curve correspond to the swelling of a ten-site strand network.

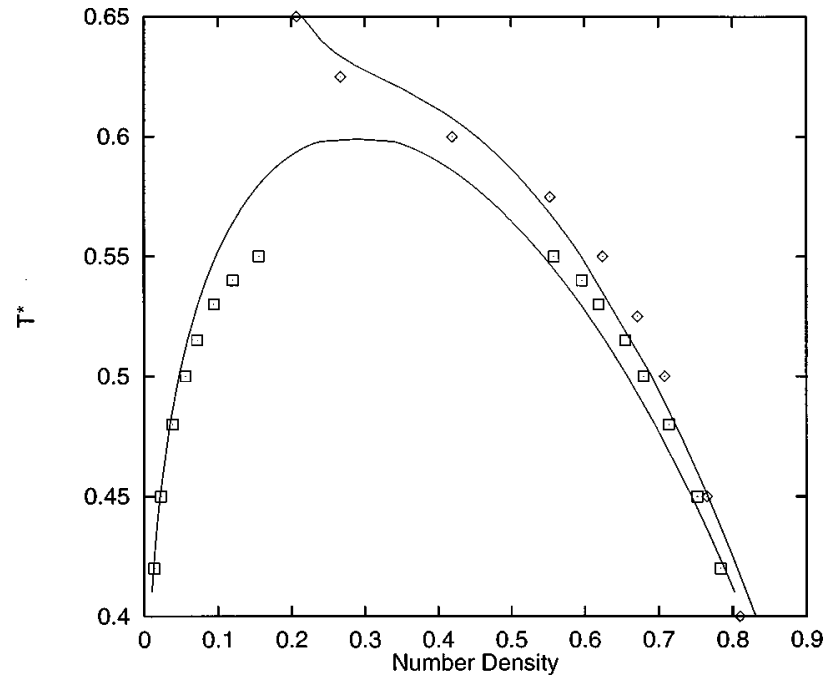

FIG. 7. Simulated vapor-liquid coexistence curve for the MLJ monomeric fluid from Gibbs ensemble simulations (squares) and temperature-density isobar at $P_{r}=1.64$ from constant pressure simulations (diamonds). The lines correspond to the predictions of Sanchez-Lacombe theory with parameters from Table I. Standard deviations of simulated points are typically of the same size as the symbols.

formed by four identical chains bonded at the ends ${ }^{15}$ ). Such pumpkin molecules were designed to match the connectivity ratio of our $N_{\mathrm{str}}=10$ network. It can be seen that the curve for the linear 42-mer is similar to that for the pumpkin molecules, but the latter curve has a lower LCST and encircles the former. As expected, the swelling curve for the gel follows closely the polymer-rich branch of the solventpumpkin system (the difference is due to the elastic pressure that slightly reduces the free volume of the gel). Incidentally, the LCST for the monomer-pumpkin system appears to agree with the temperature where the gel-swelling curve exhibits a peak.

The peculiar shape of the gel-swelling curves of SW gels shown in Fig. 3 prompted us to study a system interacting through a different potential energy model. For this purpose, we employed the modified Lennard-Jones potential (MLJ) model described in Sec. II. The coexistence curve for the pure monomeric MLJ solvent, shown in Fig. 7, was simulated by employing the Gibbs ensemble method. ${ }^{22}$ The critical properties estimated from these data are $T_{c}=0.567$ and $P_{c}=0.049$; these values are used in the remainder of this work to reduce temperatures and pressures. Figure 7 also shows the pure solvent densities along the $P_{r}=1.64$ isobar. Figure 8(a) shows results for the equilibrium swelling of gels at $P_{r}=1.64$ of three strand lengths, namely, 10, 20, and 40 sites, and one subcritical isobar at $P_{r}=0.66$ for $N_{\text {str }}=40$ (solvent's boiling point $T_{b_{r}}=0.936$ ). The simulated phase diagram of a monomer-20-mer system is also included in Fig. 8(a). The LCST for this system lies around $T_{r}=1.07$. Clearly, at $P_{r}=1.64$ the gel with longer strands swells significantly more than the short strand gel; this behavior is expected, since for a given degree of deformation (swelling), a long strand gel generally exhibits a smaller elastic pressure. ${ }^{15}$ The effect of strand length on swelling is more 


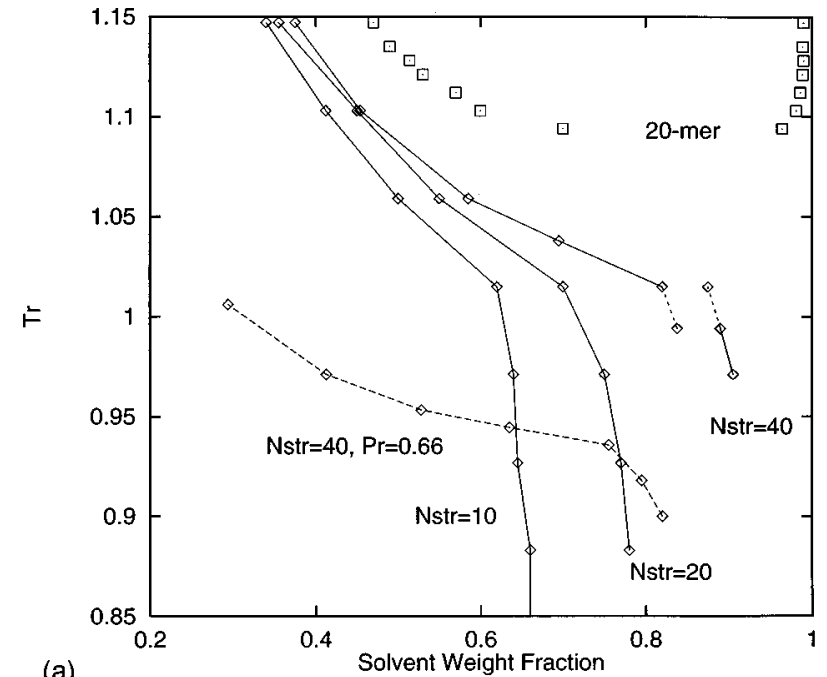

(a)

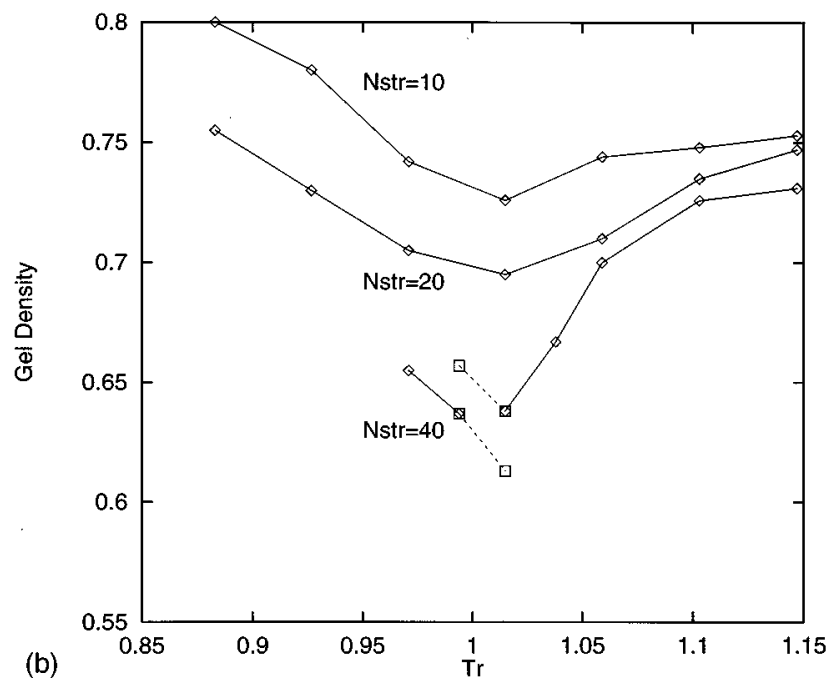

FIG. 8. Simulated isobars for MLJ systems, (a) gel-swelling and polymersolvent miscibility, and (b) gel densities. All simulated isobars correspond to $P_{r}=1.64$ (diamonds connected by straight full lines); in (a) an additional subcritical isobar at $P_{r}=0.66$ is shown by diamonds connected by dotted lines, and coexistence compositions of a monomer-20-mer system (from expanded-Gibbs ensemble simulations) are shown by squares. Gel swelling increases as the network strand $\left(N_{\text {str }}\right)$ becomes longer. For the 40 -site gel, an incipient discontinuous transition appears to take place at $T_{r} \sim 1.02$; the dotted lines in this region show the hysteresis of the trends. A small discontinuity is observed in both the gel-swelling curve (a) and the gel density curve (b). Temperature and pressure are reduced with respect to the critical properties of the pure monomeric solvent.

pronounced at subcritical temperatures and becomes smaller for high, supercritical temperatures.

Figure 8(a) should be compared with Figs. 2 and 6 for the SW system. Overall, similar trends are observed in both types of systems, namely, (i) swelling shows a decreasing trend for $T_{r}>0.8$, (ii) a marked, monotonic shrinkage of the gel occurs around the solvent's boiling temperature for $P_{r}<1$ isobar, and for $T_{r}>1.0$ for the $P_{r}>1$ isobars (around the LCST of the uncrosslinked polymer-solvent system), (iii) no evidence of a discontinuous transition is observed for short strand lengths. The main difference between the swelling curves of Figs. 6 and 8(a) is that swelling decreases very little and uniformly for the MLJ gel in the range of subcriti- cal temperatures examined here. It must be pointed out that simulations of gel swelling are plagued by ergodicity problems and slow relaxations. These problems are more serious for SW systems, where the amplitude of volume fluctuations must necessarily be small to avoid overlaps after a trial volume move. For SW and MLJ systems, the shape of the swelling curves at low temperatures could not be determined with good precision; and near the solvent's critical temperature, finite-size effects may have a pronounced effect on our results.

While gel swelling generally exhibits a significant shrinkage as it is heated above $T_{r}=1$, Fig. 8(a) shows that the shrinkage is more pronounced as the strand length increases. For the 40-site strand gel, there appears to be two branches of gel swelling around $T_{r}=1.02$; one branch corresponds to a highly swollen gel and extends to lower temperatures, and the other branch corresponds to more collapsed states and extends to higher temperatures. This suggests that the two branches must be connected by a discontinuous change in gel swelling. There is, however, some hysteresis in moving the system from one swelling branch to the other, which precludes the precise determination of the temperature where the transition takes place. Figure 8(b) shows the variation of gel density with temperature. Interestingly, all curves in Fig. 8(b) exhibit a minimum in density at around $T_{r}$ $=1.015$. This behavior can be explained as follows. At subcritical temperatures, gel density decreases upon heating because of the thermal expansion of its components; gel shrinkage is consequently mild. At supercritical temperatures, however, the gel density increases because the amount of solvent in the gel decreases significantly; the gel density approaches the pure polymer density (which is significantly higher than that of the solvent). The density minimum for the 40 -site strand gel is not only sharper than those of the other gels, but it also exhibits a noticeable discontinuity; such discontinuity is the hallmark of first-order transition. Indeed, the two distinct branches in the density versus temperature trends observed in Fig. 8(b) are consistent with the two swelling branches encountered for the same gel in Fig. 8(a).

The systematic progression of the curves in Figs. 8(a) and 8(b) for increasing strand lengths lends support to the hypothesis that gel swelling exhibits a more pronounced volume phase transition for network strands longer than 40 sites (at $T_{r}>1$ ). Unfortunately, the slow equilibration of large, highly swollen gels can trap the system in metastable states and lead to large uncertainties; the error bars near $T_{r}=1$ in Fig. 8(a) are between two and three times the size of a symbol. Consequently, the first-order character of volume phase transition is difficult to establish from simulation of long stranded gels. It is also important to bear in mind that firstorder phase transitions tend to be suppressed in simulations of finite-size systems. Finite-size effects can be particularly significant in our model gels because of the regular topology of our networks. Furthermore, density fluctuations associated with the proximity to the solvent's critical point are likely to exacerbate fluctuations of gel swelling and hamper equilibration. 


\section{COMPARISON TO THEORETICAL CALCULATIONS}

As discussed in the Introduction, a theory of gel swelling by a sub- or super critical solvent must be able to account for mixing, compressibility, and elastic effects within a gel. The approach of Marchetti et al..$^{5}$ provides perhaps the most convenient theoretical baseline; it combines widely used theories to describe the thermodynamics of compressible polymer solutions (the Sanchez and Lacombe theory ${ }^{13,14}$ ) and the network's elastic free energy (the Flory-Rehner ${ }^{12}$ ). In the theory proposed by of Marchetti et al.,$^{5}$ the pressure $P$ and the solvent's chemical potential $\mu_{s}$ in a mixture of polymer network (subscript $g$ ), solvent (subscript $s$ ), and vacancies (subscript $o$ ) are given by

$$
\begin{aligned}
-\frac{P}{k_{B} T}= & \frac{1}{v_{o}^{*}}\left[\ln \phi_{o}+\left(1-\frac{v_{o}^{*}}{v_{s}^{*}}\right) \phi_{s}+\phi_{g}\right] \\
& +\left[\left(\chi_{s o}^{\prime} \phi_{s}+\chi_{g o}^{\prime} \phi_{g}\right)\left(\phi_{s}+\phi_{g}\right)\right. \\
& \left.-\chi_{s g}^{\prime} \phi_{s} \phi_{g}\right]+\rho_{\nu}\left(\frac{\phi_{g}}{\phi_{g}^{o}}\right)^{1 / 3}, \\
\frac{\mu_{s}}{v_{s}^{*} k_{B} T}= & \frac{1}{v_{s}^{*}}\left[\ln \phi_{s}+\left(1-\frac{v_{s}^{*}}{v_{o}^{*}}\right) \phi_{o}+\phi_{g}\right] \\
& +\left[\left(\chi_{s g}^{\prime} \phi_{g}+\chi_{s o}^{\prime} \phi_{o}\right)\left(\phi_{o}+\phi_{g}\right)\right. \\
& \left.-\chi_{g o}^{\prime} \phi_{o} \phi_{g}\right]+\rho_{\nu}\left(\frac{\phi_{g}}{\phi_{g}^{o}}\right)^{1 / 3},
\end{aligned}
$$

where $v_{o}^{*}$, and $v_{s}^{*}$ are the volumes of the (empty) lattice site and the solvent molecule; $\phi_{o}, \phi_{s}$, and $\phi_{g}$ are the lattice site fractions (or volume fractions) of vacancies, solvent, and polymer, respectively; and $\rho_{\nu}$ is the density of tetrafunctional crosslinks per unit volume of gel at synthesis (where the polymer volume fraction was $\phi_{g}^{o}$, e.g., the undeformed state). The interaction parameters per lattice site are defined as

$$
\begin{aligned}
& \chi_{s o}^{\prime}=P_{s}^{*} / k_{B} T, \\
& \chi_{g o}^{\prime}=P_{g}^{*} / k_{B} T, \\
& \chi_{s g}^{\prime}=\left[P_{s}^{*}+P_{g}^{*}-2\left(1-Z_{s g}\right)\left(P_{s}^{*} P_{g}^{*}\right)^{1 / 2}\right] / k_{B} T,
\end{aligned}
$$

where $P_{s}^{*}$ and $P_{g}^{*}$ are cohesive energy densities for the pure solvent and polymer network, respectively, and $Z_{s g}$ is an adjustable parameter that characterizes the mixture $\left(k_{B}\right.$ is Boltzmann's constant). The three terms on the right-hand side of Eqs. (3) and (4) correspond to entropic mixing contribution, energetic mixing contribution, and entropic elastic effects, respectively. In the Sanchez-Lacombe theory for mixtures, the volume of a vacant lattice site need not be the same for the different species. However, in the implementation of Marchetti et al., it is assumed that there is a unique

\begin{tabular}{|c|c|c|c|}
\hline Parameter & Units & Value & Comments \\
\hline$P_{s}^{*}$ & $\epsilon / \sigma^{3}$ & 3.859 & Solvent's cohesive energy \\
\hline$v_{s}^{*}$ & $\sigma^{3}$ & 0.980 & $\begin{array}{l}\text { Close-packed vol. of solvent } \\
\text { molecule }\end{array}$ \\
\hline$v_{0}^{*}$ & $\sigma^{3}$ & 0.1508 & Volume of a solvent's mer \\
\hline$P_{g}^{*}$ & $\epsilon / \sigma^{3}$ & 4.3 & Polymer gel's cohesive energy \\
\hline$\rho_{\nu}$ & molec./ $\sigma^{3}$ & 0.016 & Unswollen cross-link density \\
\hline$Z_{s g}$ & $\cdots$ & 0.0 & Mixing correction factor \\
\hline$\phi_{0}$ & $\cdots$ & $1.12-0.43 T^{*}$ & Vol. fraction of dry polymer \\
\hline$v^{*}(20$-mer $)$ & $\sigma^{3}$ & 13.0 & 20-mer closed-packed volume \\
\hline
\end{tabular}
value of $v_{o}^{*}$; this is equivalent to assuming that the closedpacked volume of a "mer" is the same for either component in the mixture. The expression for the elastic contributions considered by Marchetti et al. also differs from that of Flory-Rehner in that only the leading terms of the elastic free energy expression are retained (network chains behave
TABLE I. Parameters for the theories of Marchetti et al. and SanchezLacombe for MLJ systems.

as Hookean springs). The prefactor $\rho_{\nu}$ can therefore be viewed as an adjustable parameter to fit select experimental data, but it is supposed to scale as $\sim N_{\text {str }}^{-1}$ (e.g., proportional to the concentration of crosslinks). The closed-packed volume fraction of component $i$ in the system $\phi_{i}^{*}$ (e.g., excluding vacancies) is related to $\phi_{i}$ by $\phi_{i}^{*}=\phi_{i} /\left(1-\phi_{o}\right)$. For our systems, $\phi_{i}^{*}$ is equivalent to a weight fraction.

Seven parameters must be specified in the above model to compute swelling equilibria: $P_{s}^{*}, v_{s}^{*}, v_{o}^{*}, P_{g}^{*}, Z_{s g}, \phi_{o}$, and $\rho_{\nu}$. The values of these parameters are chosen so as to describe select simulation data of the MLJ solvent and network; these parameters are given in Table I. The first three parameters were found by fitting saturation liquid densities and pressures for the pure solvent near $T^{*}=k_{B} T / \epsilon=0.43$ (following the procedure described by Sanchez and Lacombe $^{13}$ ). Figure 7 also shows the binodal curve and the $P_{r}=1.64$ isobar predicted by the model. As expected from a mean-field model, the phase behavior near the critical point of the solvent is not described accurately. Parameter $P_{g}^{*}$ was found by a least-squares fit of simulated density data for a $N_{\text {str }}=40$ network at $T^{*}=0.50$ and $T^{*}=0.55$. Given that a site in the polymer network is identical to a solvent molecule, we set $Z_{s g}=0.0$ (e.g., Lorentz-Berthelot mixing rules are employed for $P_{s g}^{*}$ ). Parameter $\rho_{\nu}$ was determined by matching the simulated swelling equilibria for the $N_{\text {str }}$ $=20$-gel at $T_{r}=0.88 ; \rho_{\nu}$ for other strand lengths was found by rescaling this value by a factor $20 / N_{\text {str }}$. For $\phi_{o}$ we used the polymer volume fraction for the dry network at the temperature of interest.

For a given temperature and pressure, the equilibrium swelling for a gel is calculated by first computing the chemical potential for the pure solvent $\mu_{s}$ at those conditions. Equations (3) and (4) are then solved for the two unknowns $\phi_{o}$ and $\phi_{s}\left(\phi_{g}=1-\phi_{o}-\phi_{s}\right)$. A LCST-type of binodal curve for the monomer-20-mer system is computed based on Sanchez-Lacombe's theory using the same parameters as in the solvent-gel system (except for the elastic term); an extra parameter is needed, $v^{*}(20$-mer $)$, which is estimated by assuming that $v^{*}(n-\mathrm{mer}) / v^{*}$ (monomer) $=v_{e x}^{*}(n$-mer $) / v_{e x}^{*}($ monomer $)$, where $v_{e x}^{*}(n$-mer $)$ is the volume excluded by an $n$-mer chain ${ }^{23}$ (the parameters are listed in Table I). The LCST curve calculation requires solution of the equations that equalize the chemical potentials of both species at the specified temperature and pressure.

The swelling curves based on Eqs. (3) and (4) for the 


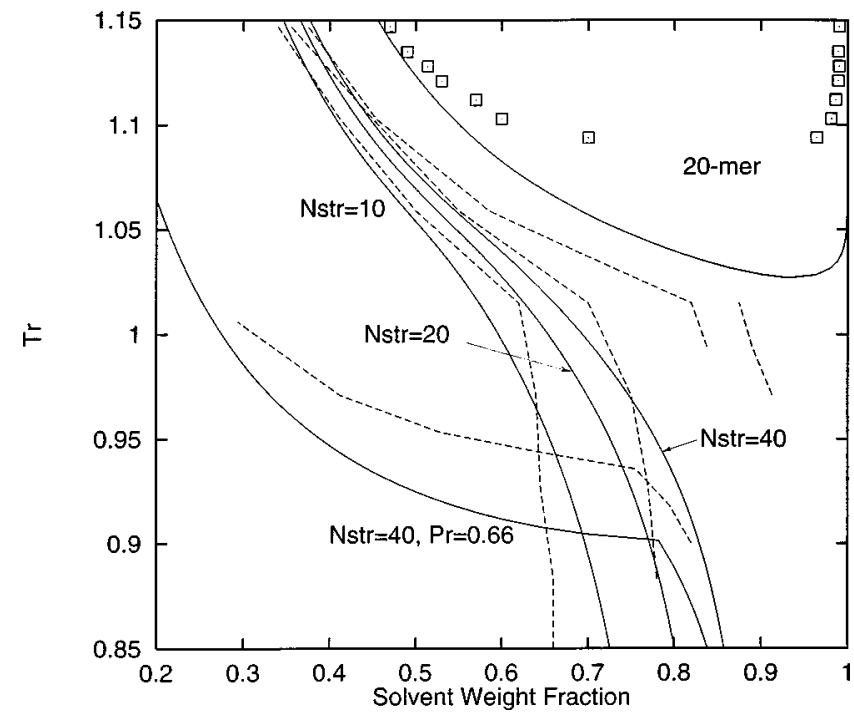

FIG. 9. Isobars for equilibrium swelling of MLJ gels as predicted by Eqs. (3) and (4), and binodal curve predicted by the Sanchez-Lacombe theory for the monomer-20-mer system (also a full line). Unless indicated otherwise, the pressure of the system is fixed at $P_{r}=1.64$. Simulated gel-swelling isobars are shown by broken lines [which pass through the data points of Fig. 8(a)], and simulated binodal data for the monomer-20-mer system are shown by squares.

MLJ gels are shown in Fig. 9 by full lines, along with the simulation data (temperatures have been reduced by the critical temperature of the pure solvent, i.e., $T_{c}=0.567$ for the simulation data, and $T_{c}=0.599$ for the lattice results). The theory is able to capture most qualitative features of the simulated curves. In particular, theoretical swelling curves for $P_{r}=1.64$ exhibit a monotonic decreasing trend as temperature is raised, and gel shrinkage is more pronounced for $T_{r}>1$, where the curves tend to follow the dense-phase branch of the LCST phase diagram of the solvent-chain system. The theoretical curve for $P_{r}=0.66$ also shows a rapid gel shrinkage as the temperature is raised above the solvent's boiling point, $T_{r}=0.885$ for the lattice solvent; interestingly, the theory predicts a discontinuity in the slope of the curve at that point. There are, however, a number of shortcomings of the theoretical model, namely,

(i) For the monomer-20-mer system, the predicted LCST is significantly lower than the simulated value.

(ii) Gel shrinkage is much more gradual around $T_{r}$ than observed in simulations.

(iii) The effect of $N_{\text {str }}$ on the degree of swelling is severely underestimated (e.g., changes in swelling are milder than those observed in simulations).

(iv) No evidence of a discontinuous phase transition is predicted for the $N_{\text {str }}=40$ gel.

The parameters employed here could perhaps be optimized to improve agreement with simulation; however, our parametric exploratory calculations appear to indicate that the theory lacks the fine-tuning capabilities required to accurately describe density changes, and the interplay between different entropic, energetic, and elastic effects; particularly in the vicinity of $T_{c}$. Given the numerous assumptions built into the theory, it is difficult to assess their relative impor- tance in accounting for the deviations from simulation data. For example, it should be pointed out that although elastic contributions are typically small (difficult to measure accurately), they play a crucial role in the swelling behavior of gels. $^{15,24}$

It has been shown by simulation ${ }^{10}$ that chain collapse (e.g., a coil-to-globule transition or CTGT) occurs in a chain-solvent system in the vicinity of a LCST. It has also been found that a discontinuous change in gel swelling can take place if the swelling curve intersects the LCST curve (near the CTGT) for the uncrosslinked polymer-solvent system. ${ }^{6}$ At subcritical pressures, the LCST for a chainsolvent system becomes the solvent's boiling point (see Fig. 1), e.g., the solvent's weight fraction at the LCST, $\phi_{s}^{*}(\mathrm{LCST})$, is unity. For supercritical pressures, $\phi_{s}^{*}$ (LCST) tends asymptotically to unity in the long chain limit. ${ }^{14}$ Consequently, the curve of gel swelling can only come near the CTGT if $\phi_{s}^{*}$ is close to unity, e.g., for high degrees of swelling. This happens as $N_{\text {str }} \rightarrow \infty$, unfortunately, $\phi_{s}^{*}$ (LCST) also comes closer to unity as $N_{\text {str }}$ increases. The theoretical calculations discussed here illustrate how these competing trends neutralize each other, while our simulation results do not clearly establish the first-order character of a gel-swelling transition near $T_{c}$. From this analysis, it can be argued that a discontinuous gel volume transition would be favored by networks with sufficiently long strands but with very low crosslink density (e.g., with a high fraction of inactive strands, trifunctional crosslinks, etc.). The pressure of the system also plays an important role (see Fig. 1); by changing the density of the solvent at any given pressure, it determines the solvent quality, the degree of swelling, the location of the CTGT and, ultimately, the occurrence of volume phase transitions.

\section{CONCLUSIONS}

The equilibrium swelling of model nonionic gel-solvent systems has been studied by Monte Carlo simulations in order to elucidate the effect of temperature and pressure on the composition and swelling of the gel. Our model gels are represented by a crosslinked network of polymer strands with perfect topology; the solvent is a simple monomeric fluid at either subcritical or supercritical conditions. Two molecular models were considered; in one case network and solvent sites interact through a square-well potential energy model and, in the other, sites experience a modified LennardJones-type potential energy.

Curves of gel swelling as a function of temperature have been generated at constant pressure. An analysis of such curves has revealed the following:

Subcritical pressures: Gel swelling exhibits a monotonic decreasing trend upon heating $\left(T_{r}>0.75\right)$, whose onset lies near the solvent's boiling point. For the short strand gels studied in this work, no evidence of a discontinuous desorption of solvent from the gel has been detected.

(ii) Supercritical pressures: For subcritical temperatures $\left(0.75<T_{r}<1\right)$, gel swelling tends to be somewhat insensitive to temperature; mild decreasing or increas- 
ing trends can occur, giving rise to a local maximum in swelling. For supercritical temperatures (at constant pressure), gel swelling typically exhibits a monotonic decreasing trend whose onset lies near the critical temperature of the pure solvent. For the MLJ gel with 40-site strands, however, gel swelling does exhibit features characteristic of an incipient discontinuous volume phase transition.

For supercritical conditions, the LCST and the polymerrich branch of the phase diagram for the solventuncrosslinked polymer system can also be used to predict gel swelling. This is particularly true when the polymer is long enough to have a compressibility similar to that of the pure network. Upon heating, gel swelling exhibits a clear decreasing trend slightly below such a LCST. For our model systems with highly simplified, nonpolar, nonionic interactions, and for the conditions studied here, first-order volume phase transitions are difficult to attain.

The theory of Marchetti et al. has been employed to calculate the swelling behavior of the simulated MLJ gels. The theory predicts swelling curves which are qualitatively consistent with our simulation results, but it underestimates considerably the changes of gel swelling brought about by changes in temperature and strand length. This theory does not predict a discontinuous swelling transition in long strand gels.

More importantly, our results for the swelling behavior of gels with different strand lengths suggest that first-order volume phase transitions will be favored by long network strands; however, other means to decrease the crosslinking density may be more effective (e.g., trifunctional crosslinks, inactive strands, etc.). It is unclear, however, what the effect of pressure is in promoting discontinuous transitions. Additional (unreported) simulations have explored the effect of a small chemical mismatch in polymer-solvent interactions for $N_{\text {str }}=20 \mathrm{gels}$; these simulations failed to detect a temperature-driven volume phase transition. Our study has been limited to a rather idealized situation in which polymer sites and solvent have the least asymmetry (in both size and strength of interactions). The presence of anisotropic interactions between the solvent and different parts of the polymer network (e.g., due to the presence of side groups, stiffer chain sections, or to the formation of hydrogen bonds) could lead to a rich and complex swelling behavior. The results obtained in this work provide a reference point for future microscopic studies on the thermodynamics of gelsupercritical fluid systems; simulation of increasingly complex models can then help elucidate the effects of specific chemical details on the swelling behavior of realistic gels.

\section{ACKNOWLEDGMENTS}

Financial support from the National Science Foundation (Grant No. CTS-9629135) is gratefully acknowledged. One of the authors (J.J.dP.) is also grateful to the Camille and Henry Dreyfus Foundation for a Teacher-Scholar Award.

${ }^{1}$ B. Erman and J. E. Mark, Structures and Properties of Rubberlike Networks (Oxford, NY, 1997).

2 Adv. Polym. Sci. 109-110, (1993).

${ }^{3}$ K. Dusek and D. Patterson, J. Polym. Sci., Part A: Polym. Chem. 6, 1209 (1968).

${ }^{4}$ Y. Hirokawa, T. Tanaka, and E. S. Matsuo, J. Chem. Phys. 81, 6379 (1984).

${ }^{5}$ M. Marchetti, S. Prager, and E. L. Cussler, Macromolecules 23, 1760 (1760).

${ }^{6}$ M. Marchetti, S. Prager, and E. L. Cussler, Macromolecules 23, 3445 (1990).

${ }^{7}$ R. F. S. Freitas and E. L. Cussler, Sep. Sci. Technol. 22, 911 (1987).

${ }^{8}$ E. Kiran, in Supercritical Fluids. Fundamentals for Applications, edited by E. Kiran and J. M. H. Levelt Sengers (Kluwer Academic, Dordrecht, NATO ASl Series E, 1994), Vol. 273.

${ }^{9}$ S. J. Chen, I. G. Economou, and M. Radosz, Macromolecules 25, 4987 (1992).

${ }^{10}$ G. Luna-Barcenas, J. C. Meredith, I. C. Sanchez, K. P. Johnston, D. G. Gromov, and J. J. de Pablo, J. Chem. Phys. 107, 10782 (1997).

${ }^{11}$ D. G. Gromov, J. J. de Pablo, G. Luna-Barcenas, I. C. Sanchez, and K. P. Johnston, J. Chem. Phys. 108, 4647 (1998).

${ }^{12}$ P. J. Flory and J. Rehner, Jr., J. Chem. Phys. 11, 521 (1943); P. J. Flory, Principles of Polymer Chemistry (Cornell University Press, New York, 1953).

${ }^{13}$ I. C. Sanchez and R. H. Lacombe, J. Phys. Chem. 80, 2353 (1976); R. H. Lacombe and I. C. Sanchez, ibid. 80, 2568 (1976).

${ }^{14}$ I. C. Sanchez and R. H. Lacombe, Macromolecules 11, 1145 (1978).

${ }^{15}$ F. Escobedo and J. J. de Pablo, J. Chem. Phys. 106, 793 (1996).

${ }^{16}$ F. Escobedo and J. J. de Pablo, Mol. Phys. 90, 437 (1997).

${ }^{17}$ F. A. Escobedo and J. J. de Pablo, J. Chem. Phys. 104, 4788 (1996).

${ }^{18}$ L. Vega, E. de Miguel, and L. F. Rull, J. Chem. Phys. 96, 2296 (1992).

${ }^{19}$ A. Yethiraj and C. K. Hall, J. Chem. Phys. 95, 8494 (1991).

${ }^{20}$ K. K. Lee, E. L. Cussler, M. Marchetti, and M. McHugh, Chem. Eng. Sci. 45, 766 (1990).

${ }^{21}$ The densities of the network for other pressures (not too different from zero) are only slightly different from those at $P_{r}=0$.

${ }^{22}$ A. Z. Panagiotopoulos, Mol. Phys. 61, 813 (1987).

${ }^{23}$ M. Denlinger and C. K. Hall, Mol. Phys. 71, 541 ().

${ }^{24}$ J-U. Sommer, T. A. Vilgis, and G. Heinrich, J. Chem. Phys. 100, 9181 (1994); H. L. Trautenberg, J-U. Sommer, and D. Goritz, J. Chem. Soc., Faraday Trans. 91, 2649 (1995); J-U Sommer, M. Shulz, and H. L. Trautenberg, J. Chem. Phys. 98, 7515 (1993). 